\title{
Estudio descriptivo del cribado de cromosomopatías en el primer trimestre de la gestación, en el Hospital Clínico San Carlos de Madrid, España
}

\author{
Tamara Illescas M. ${ }^{1}$, Pluvio Jesús Coronado M. ${ }^{2}$, María Dolores Ortega H. ${ }^{3}$, \\ Patricia Soler R. 2, Gloria Costa M. 2, Joaquín Montalvo M. 2 \\ 1 Clínica Delta. ${ }^{2}$ Servicio de Ginecología y Obstetricia, ${ }^{3}$ Servicio de Análisis Clínicos, Hospital Clínico San Carlos. \\ Madrid, España.
}

\section{RESUMEN}

Antecedentes: El cribado combinado de cromosomopatías, fundamentalmente trisomía 21, en el primer trimestre de gestación, se introdujo en los años '90 y está basado en un cálculo del riesgo a través de la combinación de la edad materna, la translucencia nucal fetal (TN), y los marcadores bioquímicos maternos (PAPP-A y fBHCG). Mediante esta combinación de marcadores se obtiene una sensibilidad para aneuploidías del $85-90 \%$, con una tasa de falsos positivos (FP) del $5 \%$. Objetivo: El propósito de este trabajo es describir la población cribada y analizar los resultados desde la implantación de la técnica en nuestro centro. Método: Estudio observacional de la población gestante que acudió para cribado de cromosomopatías durante el primer trimestre de la gestación al Hospital Clínico San Carlos, desde julio de 1999 hasta diciembre de 2009. Resultados: El tamaño muestral fue de 21.194 gestaciones simples, con un $12,5 \%$ de mujeres con más de una gestación y 141 casos de aneuploidía $(6,6 \%$ ). Se describe las características de la población y el comportamiento de las variables estudiadas. La sensibilidad del cribado combinado fue del $70 \%$, con una tasa de FP de 2,3\%. Conclusión: La tasa de detección del cribado combinado de cromosomopatías en el primer trimestre de gestación, es menor que la publicada en la literatura, aunque ha ido mejorando con los años, probablemente por una mejor formación de los ecografistas. En contraposición, la tasa de FP es muy baja.

\section{PALABRAS CLAVE: Cribado, aneuploidías, primer trimestre, gestación}

\section{SUMMARY}

Background: Screening for aneuploidies, mainly trisomy 21, during the first trimester of gestation, was introduced in the '90s and is based on a calculation of the risk through the combination of maternal age, nuchal translucency (NT) and biochemical parameters (PAPP-A and $\mathrm{ABHCG).} \mathrm{By} \mathrm{means} \mathrm{of} \mathrm{this} \mathrm{combination} \mathrm{we} \mathrm{can}$ obtain a detection rate for aneuploidies of $85-90 \%$, with a false positive rate of $5 \%$. Objective: To describe our population and analyze our results related to combined screening for aneuploidies, during the period of time it has been performed in our hospital. Methods: Observational study of all pregnant women who attended Hospital Clínico San Carlos for screening of chromosomopaties during first trimester of gestation, from July 1999 to December 2009. Results: Sample size was 21,194 single pregnancies, with $12,5 \%$ of women with more than one gestation, and 141 cases of aneuploidy $(6,6 \%)$. We describe the characteristics of our 
population and the distribution of the parameters studied. Combined screening had a detection rate of $70 \%$ with a false positive rate of 2,3\%. Conclusion: Screening for aneuploidies during first trimester of gestation, in Hospital Clínico San Carlos, has a lower detection rate than previously reported. However, the false positive rate is very low.

\section{KEY WORDS: Screening, aneuploidies, first trimester, pregnancy}

\section{INTRODUCCIÓN}

El diagnóstico prenatal de cromosomopatías requiere de técnicas invasivas de alto coste, que implican cierto riesgo de pérdida fetal, y que deben ser realizadas por personal especializado.

Desde que se planteó la necesidad de un cribado poblacional, para la detección de individuos con alto riesgo de cromosomopatía, se ha diseñado diferentes estrategias, cada una de las cuales gana en complejidad para mejorar en sensibilidad (S), con una tasa de falsos positivos (FP) aceptable.

El cribado combinado de cromosomopatías, fundamentalmente para trisomía 21, en el primer trimestre de gestación, se introdujo en los años '90 y está basado en un cálculo del riesgo a través de la combinación de la edad materna, la translucencia nucal fetal (TN), y los marcadores bioquímicos maternos como la fracción libre de la cadena $B$ de la gonadotropina coriónica ( $\mathrm{f} B \mathrm{HCG}$ ) y la proteína plasmática asociada al embarazo (PAPP-A) (1).

Mediante esta combinación de marcadores se obtiene una sensibilidad del $85-90 \%$, con una tasa de FP del $5 \%$. Aunque está diseñado con algoritmos específicos para trisomías 21 y 18, detecta además el $80 \%$ de las trisomías del cromosoma 13 , el $96 \%$ de los síndromes de Turner y el $62 \%$ de las demás aneuploidías sexuales (2).

Los marcadores TN, PAPP-A, fBHCG son independientes de la edad materna $(3,4)$, requisito necesario para poder combinarlos en un algoritmo multivariante de riesgo. Tampoco se halló asociación entre marcadores bioquímicos y ultrasonográficos (5). La introducción de parámetros bioquímicos en el cribado aumenta la tasa de detección en un $16 \%$, respecto a la sensibilidad del cribado basado únicamente en la edad materna y la TN (3). Para el cálculo del riesgo, se multiplica el riesgo a priori obtenido por la edad materna, por las razones de probabilidad obtenidas a partir de las desviaciones de la medida de la TN, la fBHCG, y la PAPP-A, de sus respectivas medianas.

El objetivo de este trabajo es realizar un estudio descriptivo, desde su implantación, del cribado combinado de cromosomopatías en el primer trimestre de la gestación en el Hospital Clínico San Carlos de Madrid.

\section{MATERIAL Y MÉTODOS}

Estudio observacional retrospectivo de toda la población gestante del Área Sanitaria 7 de la Comunidad de Madrid, que ha acudido para cribado de cromosomopatías durante el primer trimestre de la gestación al Hospital Clínico San Carlos, desde la instauración de la técnica en julio de 1999, hasta diciembre de 2009. Se estima que nuestra muestra $(n=21.194)$ corresponde a más del $80 \%$ de la población total del área.

Se incluyó a pacientes con gestaciones simples, con feto vivo y edad gestacional entre 11 y 13+6 semanas (longitud cráneo-caudal o CRL entre 45 y $84 \mathrm{~mm}$ ), confirmada por ultrasonido. Con la base de datos original, se analiza y describe las características de la población y del método de cribado utilizado. Los valores de expresan en promedio, mediana, desviación estándar $( \pm D E)$, y rango intercuatílico (rango IC). Se calcula la sensibilidad (S) y la especificidad (E) de la metodología de cribado.

\section{RESULTADOS}

En el periodo analizado se realizó cribado combinado de cromosomopatías en el primer trimestre a 21.363 pacientes con gestación simple en curso. Tras excluir 169 casos por errores en el procesamiento de datos, quedó una muestra de 21.194. La distribución de las pacientes cribadas en cada uno de los años se muestra en la Figura 1.

El cribado consistió en ecografía entre las semanas 11 y $13+6$, con medida de la CRL y de la TN; analítica de sangre, el mismo día de la ecografía, para determinación de los parámetros bioquímicos PPAP-A y fBHCG; y cálculo del riesgo combinado considerando el riesgo a priori por la edad materna, el calculado a partir de la medida de la TN y (con las correcciones pertinentes según peso materno, hábito tabáquico o presencia de diabetes) el obtenido de los marcadores bioquímicos. Se consideró un resultado de riesgo aumentado de cromosomopatía cuando el riesgo combinado fue $\geq 1 / 270$, y en tal caso se ofrecía a la paciente una prueba de diagnóstico genético prenatal. 


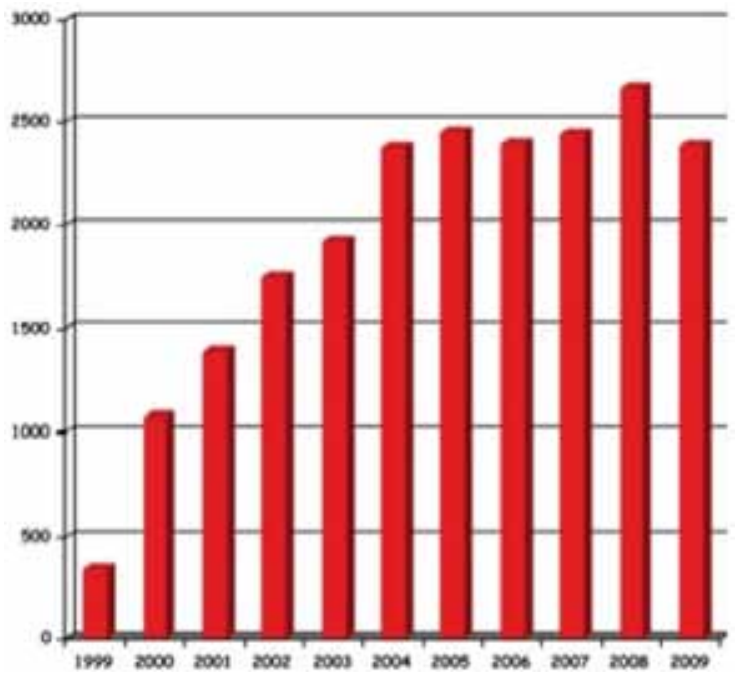

Figura 1. Pacientes cribadas cada año (1999 2009).

Las características demográficas de la población se presentan en la Tabla I; la distribución de los valores de PAPP-A, fßHCG, TN y sus MoM (múltiplos de la mediana), en la Tabla II.

La distribución por razas fue la siguiente: blanca $70,7 \%$, negra $0,7 \%$, amarilla $1,3 \%$, otras $9,1 \%$, desconocida $18,2 \%$. El $15,9 \%$ de las pacientes cribadas eran fumadoras. El $2,4 \%$ o consta como dia- béticas. El número de gestaciones cribadas y las medianas de los valores de PAPP-A, fBHCG y TN, en cada semana de gestación, se presentan en las Figuras 2 a 5.

Entre toda la población estudiada, hubo un total de 2.230 técnicas de diagnóstico prenatal $(10,5 \%$ de pruebas invasivas, bien amniocentesis o biopsia corial) y 141 fetos aneuploides (6,6\%o), de los cuales 14 fueron diagnosticados por estudio de cariotipo después del nacimiento. De las cromosomopatías, 73 fueron casos de trisomía 21 .

De los 14 fetos diagnosticados después del nacimiento $(9,9 \%$ del total de aneuploides), 13 eran casos de trisomía 21, y un caso de deleción autosómica. Cuatro de ellos tuvieron un test de cribado de alto riesgo.

De los 127 fetos aneuploides diagnosticados prenatalmente, 32 tenían un resultado de la prueba de cribado combinado de bajo riesgo, luego la técnica invasiva se realizó por otros motivos. Entre ellos, 17 (53\%) tuvieron un resultado de riesgo alto, basado en el cálculo por edad + bioquímica.

En nuestra población, teniendo en cuenta sólo la edad, el 33,3\% de las pacientes (6.911) tendrían un riesgo $\geq 1 / 270$, que correspondía a 33 años de edad. En este grupo de pacientes había 90 fetos con cromosomopatía, lo cual suponía un $63,8 \%$ del total de fetos aneuploides. De ellos, 52 eran fetos con trisomía 21 (71,2\% del total de fetos con síndrome de Down).

Tabla I

DATOS GENERALES DE LA MUESTRA EN ESTUDIO

\begin{tabular}{lcccc}
\hline & Media & Mediana & \pm DE & Rango IC \\
\hline Edad materna (años) & 30,6 & 31 & 5,3 & $(27-34)$ \\
Peso materno $(\mathrm{kg})$ & 62,5 & 61 & 11 & $(55-68)$ \\
Edad gestacional en cribado (sem) & 11,8 & 12 & 0,86 & $(11,3-12,4)$ \\
Edad gestacional en parto (sem) & 38,5 & 39 & 4,75 & $(38-40)$ \\
Peso del recién nacido (g) & 3.201 & 3.210 & 521 & $(2.910-3.545)$ \\
\hline
\end{tabular}

Tabla II

DISTRIBUCIÓN DE LOS VALORES DE PAPP-A, fßHCG, TN Y SUS MoM

\begin{tabular}{lcccc}
\hline & Media & Mediana & \pm DE & Rango IC \\
\hline PAPP-A (mUI/L) & $3.704,5$ & $3.061,0$ & $2.884,1$ & $(1.967-4.590)$ \\
MoM PAPP-A & 1,29 & 1,13 & 0,90 & $(0,78-1,60)$ \\
fBHCG (UI/L) & 41,7 & 33,2 & 37,10 & $(22,0-51,6)$ \\
MoM fBHCG & 1,23 & 1,0 & 1,09 & $(0,67-1,51)$ \\
TN (mm) & 1,31 & 1,2 & 0,61 & $(1-1,5)$ \\
MoM TN & 0,86 & 0,80 & 0,4 & $(0,67-0,97)$ \\
\hline
\end{tabular}




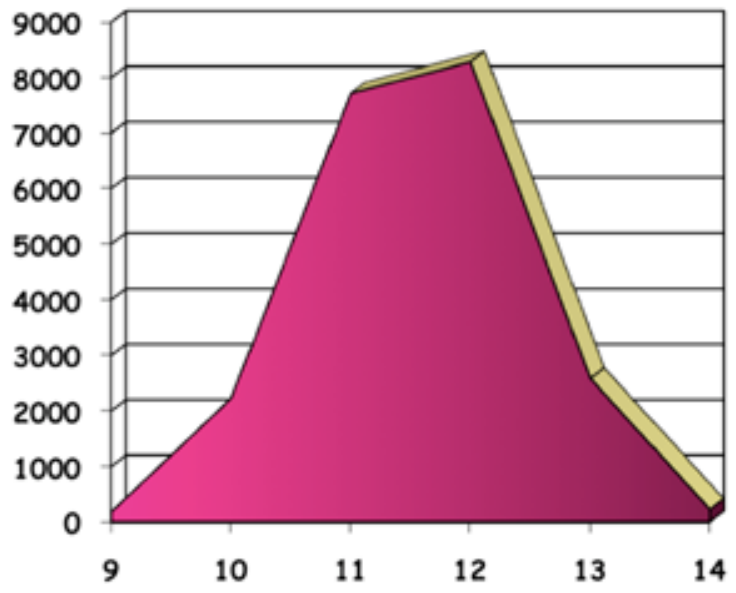

Figura 2. Número de casos cribados en cada semana cumplida de gestación

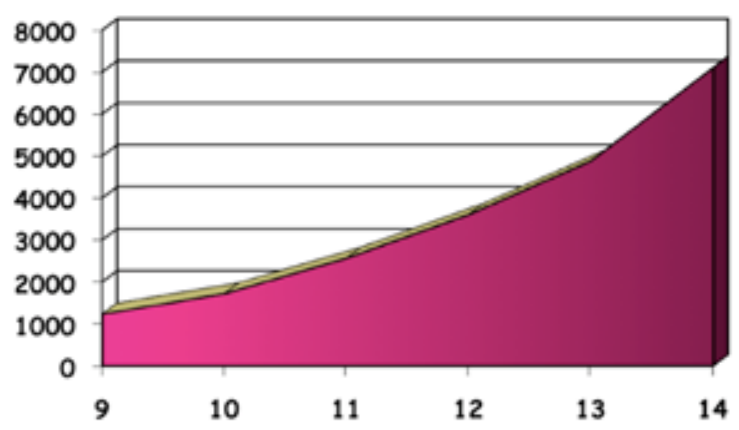

Figura 3. Mediana de los valores de PAPP-A en $\mathrm{mUl} / \mathrm{L}$, en cada semana de gestación cumplida.

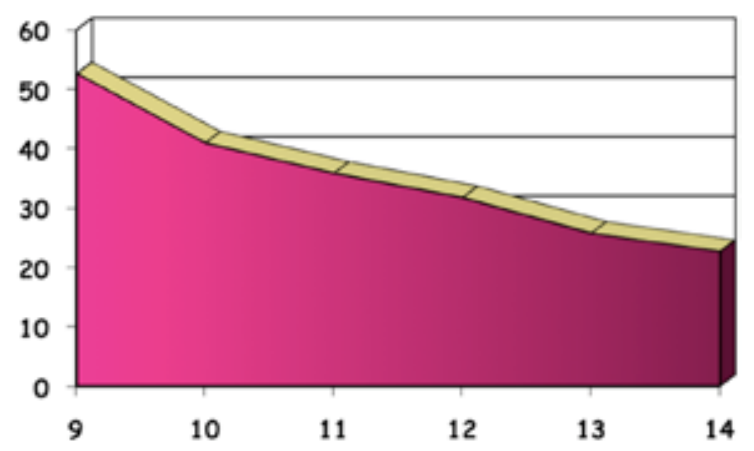

Figura 4. Mediana de los valores de fßHCG en UI/L, en cada semana de gestación cumplida.

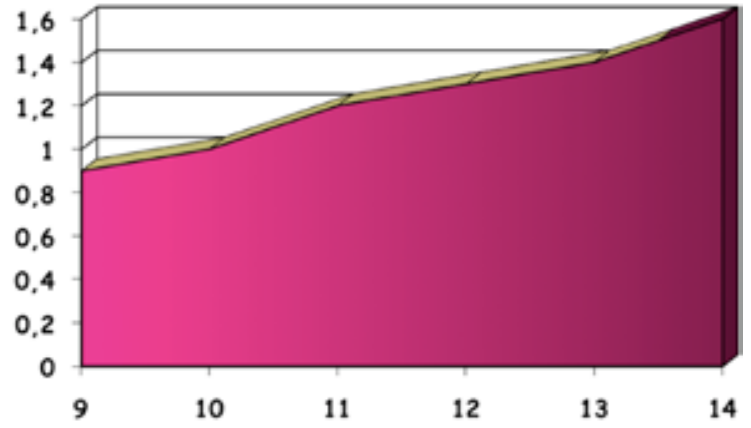

Figura 5. Mediana de los valores de TN en milímetros, en cada semana de gestación cumplida.

Respecto al análisis del riesgo combinado en nuestra población, el cálculo de la sensibilidad (S) y de la especificidad $(E)$ resulta:

$\mathrm{S}=\mathrm{VP} /(\mathrm{VP}+\mathrm{FN}) \rightarrow 99 /(99+42)=0,70$ (70\%).

$E=V N /(V N+F P) \rightarrow 20127 /(20127+465)=$ 0,9774 (97,7\%).

Por tanto, la tasa de FN (1 - S) es del $30 \%$, y la de FP $(1-E)$ es del 2,3\%.

La sensibilidad específica para trisomía 21 fue del $72,6 \%$ (se detectó 53 de los 73 fetos portadores), con una tasa de FP del $2,3 \%$. Respecto a la combinación de edad + marcadores bioquímicos, la sensibilidad es del $71 \%$, la especificidad del $84 \%$, la tasa de FN del $29 \%$ y la de FP del $16 \%$.

Se consiguió detectar, mediante este método, 20 de los 21 fetos con trisomía 18 y 6 de los 11 fetos con trisomía 13, lo que supone una sensibilidad global del 81,25\%, y una tasa de detección del 95,2\% para síndrome de Edwards y del 54,5\% para síndrome de Patau.

La sensibilidad en cada año del periodo de cribado, para el combinado y el de edad + bioquímica, se presenta en la Figura 6.

Hubo 2.343 mujeres con más de una gestación cribada en el primer trimestre (de esta manera, hubo un total de 18.684 pacientes cribadas en nuestra muestra, con un $12,5 \%$ de pacientes con más de una gestación). Estas mujeres cursaron un total de 4.852 gestaciones, de manera que 2.191 tuvieron dos, 138 tuvieron tres, 13 pacientes tuvieron cuatro, y una paciente tuvo cinco cribados. Entre estas pacientes con más de una gestación, hubo 53 diagnósticos de aneuploidía. 


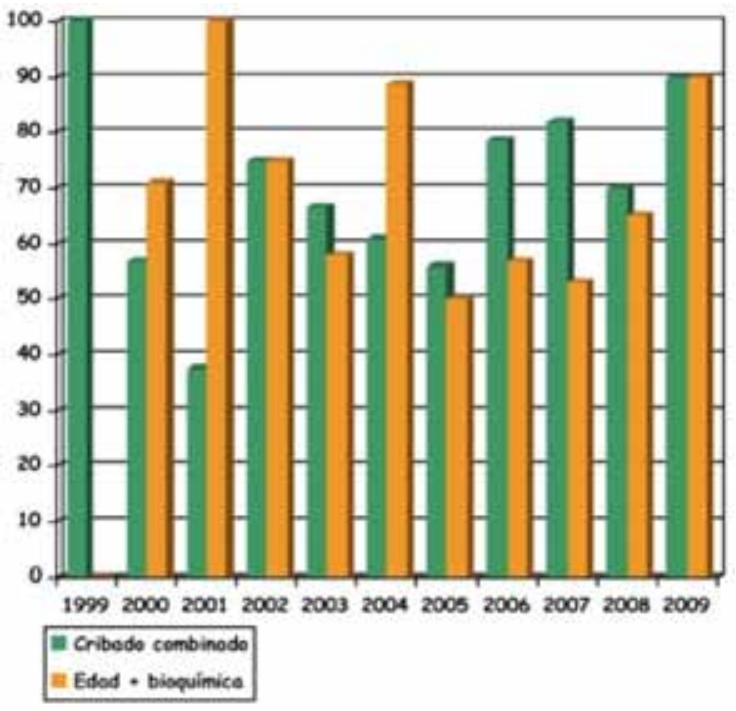

Figura 6. Distribución de la sensibilidad en cada año del periodo de cribado, para la estrategia combinada y para el cribado por edad + bioquímica.

\section{DISCUSIÓN}

El cribado combinado de cromosomopatías en el primer trimestre de la gestación se ha generalizado en muchos países, incluida España, en los últimos diez años. El Hospital Clínico San Carlos fue uno de los primeros en implantarlo en la Comunidad de Madrid. Ello permite disponer de una amplia casuística, cuyo análisis es el objeto de este trabajo.

Desde la implantación del cribado en 1999, el número de pacientes que se acoge a esta estrategia va aumentando hasta hacerse estable en 2004, año a partir del cual se criba en torno a 2.400 pacientes por año.

La edad materna media (y la mediana) de las pacientes está por encima de los 30 años, en concordancia con la tendencia social desde hace unos años de retrasar el momento de la maternidad. Además, aunque en nuestra muestra no se introduce factor de corrección en función de la forma de concepción, recordamos la reciente aparición y progresivo desarrollo de la Unidad de Reproducción Asistida en el Hospital Clínico San Carlos, cuyas pacientes han podido contribuir a un aumento de la edad media materna.

La clasificación por razas que permite el programa informático de cribado, no refleja la situación real de nuestra población. Este programa se adapta mejor a la distribución en los países anglosajones, con un porcentaje significativo de pacientes de raza negra y amarilla. Sin embargo, nuestra población tiene un porcentaje significativo de pacientes de los países del norte de África y de Latinoamérica, con diversos grados de mestizaje que no pueden ser reflejados, ya que por la clasificación disponible, se ha optado por incluir a todas estas pacientes en el grupo de raza blanca.

La mayoría de las pacientes fueron cribadas entre las 11 y 12 semanas, cumplidas de gestación. Esto sigue la tendencia generalizada a realizar el examen ecográfico en torno a la semana 12, ya que facilita la realización de una valoración anatómica precoz que complementa el propósito de cribado de aneuploidías de la prueba $(6,7)$.

La tendencia de los marcadores respecto a la edad gestacional confirma el comportamiento esperado en función de la biología $(5,7,8,9)$. De esta manera, observamos en nuestra población que, conforme avanza la edad gestacional:

- los valores de PAPP-A aumentan.

- los valores de $\mathrm{fBHCG}$ disminuyen.

- la medida de la TN aumenta.

Se realizó una técnica de diagnóstico genético prenatal (amniocentesis o biopsia de vellosidades coriales) en el $10,5 \%$ de la población. La aneuploidía más frecuente es la trisomía 21, que representa más de la mitad de los casos.

De los diagnósticos postnatales, 4 de ellos estaban en rango de alto riesgo; es de suponer que la paciente declinó el ofrecimiento de diagnóstico invasivo por razones personales o miedo a complicaciones de la prueba.

De los 10 falsos negativos de la prueba, que no se logró identificar antes del nacimiento, 6 tenían riesgos que actualmente definimos como intermedios (entre $1 / 270$ y $1 / 1000$ ), que en la actualidad comienza a manejarse con una actitud de reevaluación ecográfica (con marcadores como el hueso nasal, la valoración de la onda A en ductus venoso, y la presencia de regurgitación tricuspídea) para reajustar el riesgo e incluir a las pacientes definitivamente en la categoría de riesgo alto o bajo, con la actitud consecuente. Esta reevaluación ecográfica es una estrategia actualmente en progresiva implantación.

La tasa de detección teniendo en cuenta solamente la edad materna es baja, y similar a la referida en la literatura. La sensibilidad es algo mayor para síndrome de Down que para todas las cromosomopatías en general, probablemente debido a la clara asociación entre edad materna e incidencia de gestaciones con trisomía 21, mientras que no todas las cromosomopatías tienen la misma relación directa con la edad materna. 
La sensibilidad global del cribado, teniendo en cuenta sólo la edad y la bioquímica, es $71 \%$, similar a la de la literatura y a la obtenida en nuestra población mediante cribado combinado. La diferencia está en que, para una tasa de detección similar, los FP aumentan de un $2,3 \%$ con cribado combinado, a un $16 \%$ con edad + bioquímica.

Hay otros $32 \mathrm{FN}$ de la prueba de cribado combinado, pero fueron detectados prenatalmente mediante estudio de cariotipo. El motivo por el que se realizó una prueba de diagnóstico invasivo a estas pacientes se desconoce, pero el $53 \%$ de estas pacientes tuvo alto riesgo respecto a edad + parámetros bioquímicos.

Otros motivos para la indicación de un cariotipo antenatal (un valor de TN > p95 para su CRL, antecedentes personales o familiares de la paciente, ansiedad materna, otro tipo de marcadores ecográficos de aneuploidías) finalmente resultaron en una sensibilidad del $93 \%$, con una tasa de FP, equivalente al número de técnicas de diagnóstico prenatal realizadas con resultado normal (hubo 127 con resultado de aneuploidía) del 9,9\%.

La sensibilidad global del cribado combinado es menor que en algunos de los estudios publicados, aunque estos suelen estar referidos a una tasa de FP del 5\%, y la nuestra es del 2,3\%. La sensibilidad para trisomía 21, exclusivamente, es similar a la tasa de detección global.

Por una cromosomopatía diagnosticada prenatalmente, se ofreció una prueba invasiva (indicada por cribado combinado de alto riesgo) a casi $5 \mathrm{mu}$ jeres que gestaban un feto sano. Es un dato favorable, que indica un alto rendimiento de las pruebas de diagnóstico invasivo, gracias a la baja tasa de FP. Snijders y cols (10), refieren en un estudio sobre el cribado mediante edad materna y TN, la necesidad de 30 pruebas invasivas para diagnosticar un feto afecto de aneuploidía. No se trataba de la misma técnica de cribado, pero con este dato, y pese a que la TN es un potente marcador de forma aislada, podemos hacernos una idea en que medida la introducción de los marcadores bioquímicos puede mejorar el cribado de aneuploidías.

El riesgo de trisomía 13 y 18 se calcula sólo con variables bioquímicas, con una alta tasa de detección para síndrome de Edwards (95,2\%), y mucho más débil para síndrome de Patau (54,5\%). Sin embargo, a la detección de estas aneuploidías contribuye su frecuente asociación a hallazgos ecográficos de alta expresividad que permiten establecer muchas veces la sospecha diagnóstica. De hecho, todos los casos fueron diagnosticados prenatalmente.

En nuestra población, las medianas de los MoM de PAPP-A y fBHCG se sitúan muy próximas a 1 , lo cual indica que la población de referencia, usada por el programa informático, se aproxima mucho a nuestra muestra. Sin embargo, en el caso de la TN, la mediana de sus MoM es 0,80 . La medida de la TN es más dependiente del observador que las determinaciones analíticas y requiere una curva de aprendizaje. Además, hay varios ecografistas y esto aumenta la dispersión de los resultados. La aparente tendencia global, a subestimar ligeramente el valor de la TN, repercutiría en una determinación de riesgos menores, que conllevaría una menor sensibilidad de la prueba de cribado. Al observar la gráfica por años, la tendencia de la sensibilidad es ascendente, probablemente por una mayor formación de los ecografistas.

El estudio multicéntrico de Schielen y cols (11), mostró, para un punto de corte de alto riesgo de $1 / 250$ en el cribado combinado del primer trimestre, una sensibilidad del $71 \%$, con una tasa de FP del $4,7 \%$. También ellos hallaron, en la correcta medición de la TN, una gran posibilidad de mejoría del rendimiento de la prueba.

\section{CONCLUSIÓN}

La tasa de detección del cribado combinado de cromosomopatías en el primer trimestre de gestación, en el Hospital Clínico San Carlos, es menor que la publicada en la literatura, aunque ha ido mejorando con los años, probablemente por una mejor formación de los ecografistas. En contraposición, la tasa de falsos positivos es muy baja.

\section{BIBLIOGRAFÍA}

1. Kagan KO, Wright D, Baker A, Sahota D, Nicolaides $\mathrm{KH}$. Screening for trisomy 21 by maternal age, fetal nuchal translucency thickness, free beta-human chorionic gonadotropin and pregnancy-associated plasma protein-A. Ultrasound Obstet Gynecol 2008;31:618-24.

2. Programa para el screening de síndrome de Down. SsdwLab V 4.1. 2001.

3. Spencer K, Souter V, Tul N, Snijders R, Nicolaides $\mathrm{KH}$. A screening program for trisomy 21 at $10-14$ weeks using fetal nuchal translucency, maternal serum free B-human chorionic gonadotropin and pregnancyassociated plasma protein-A. Ultrasound Obstet Gynecol 1999;13:231-7.

4. Spencer K, Ong C, Liao W, Nicolaides KH. The influence of parity and gravidity on first trimester markers of chromosomal abnormality. Prenat Diagn 2000;20:792-4.

5. Snijders R, Johnson S, Sebire N, Noble P, Nicolaides $\mathrm{KH}$. First-trimester ultrasound screening for chromosomal defects. Ultrasound Obstet Gynecol 1996;7:21626.

6. Screening de cromosomopatías fetales. Documentos de Consenso. SEGO 2000. 
7. Bindra R, Heath V, Liao A, Spencer K, Nicolaides KH. One-Stop clinic for assessment of risk for trisomy 21 at 11-14 weeks: a prospective study of 15030 pregnancies. Ultrasound Obstet Gynecol 2002;20:219-25.

8. Herrera E. Biología molecular y bioquímica fisiológica. Segunda Ed. Madrid: Mc Graw-Hill Interamericana de España, 1991:1154-5.

9. Guyton AC, Hall JE. Tratado de Fisiología Médica. Novena Ed. Madrid: Mc Graw-Hill Interamericana de España, 1996:1138-9.
10. Snijders R, Noble P, Sebire N, Souka A, Nicolaides $\mathrm{KH}$. UK multicentre Project on assessment of risk of trisomy 21 by maternal age and fetal nuchaltranslucency thickness at 10-14 weeks of gestation. Fetal Medicine Foundation First Trimester Screening Group. Lancet 1998;542(9125):343-6.

11. Schielen PC, van Leeuwen-Spruijt M, Belmouden I, et al. Multi-centre first-trimester screening for Down syndrome in the Netherlands in routine clinical practice. Prenat Diagn 2006;26:711-8. 